NA

NASA Technical Memorandum 106150 164786 AIAA-92-3070

\title{
Increased Heat Transfer to Elliptical Leading Edges Due to Spanwise Variations in the Freestream Momentum: Numerical and Experimental Results
}

\section{D.L. Rigby}

Sverdrup Technology, Inc.

NASA Lewis Research Group

Brook Park, Ohio

and

G.J. Van Fossen

National Aeronautics and Space Administration

Lewis Research Center

Cleveland, Ohio

Prepared for the

28th Joint Propulsion Conference and Exhibit

cosponsored by the AIAA, SAE, ASME, and ASEE

Nashville, Tennessee, July 6-8, 1992

(NASA-TM-106150) INCREASED HEAT

TRANSFER TO ELLIPTICAL LEADING

EDGES DUE TO SPANWISE VARIATIONS IN

N/SA
THE FREESTREAM MOMENTUM: NUMERICAL

AND EXPERIMENTAL RESULTS (NASA)

$13 \mathrm{p}$
N93-27020

Unclas

G3/34 0164786 
... . . . . . . . . .

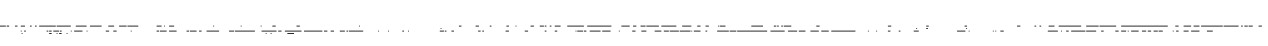

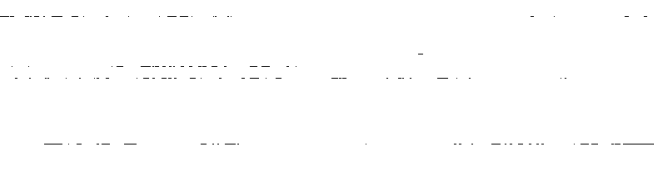

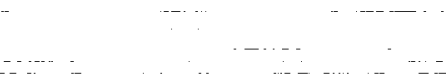

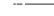


INCREASED HEAT TRANSFER TO ELLIPTICAL LEADING EDGES DUE TO

SPANWISE VARIATIONS IN THE FREESTREAM MOMENTUM:

NUMERICAL AND EXPERIMENTAL RESULTS

\author{
D.L. Rigby" \\ Sverdrup Technology, Inc. \\ Lewis Research Center Group \\ Brook Park, Ohio 44142 \\ and \\ G.J. Van Fossen \\ National Aeronautics and Space Administration \\ Lewis Research Center \\ Cleveland, Ohio 44135
}

\begin{abstract}
$\underline{\text { Abstract }}$
A study of the effect of spanwise variation in momentum on leading edge heat transfer is discussed. Numerical and experimental results are presented for both a circular leading edge and a 3:1 elliptical leading edge. Reynolds numbers in the range of 10,000 to 240,000 based on leading edge diameter are investigated. The surface of the body is held at a constant uniform temperature. Numerical and experimental results with and without spanwise variations are presented. Direct comparison of the two-dimensional results, that is, with no spanwise variations, to the analytical results of Frossling is very good. The numerical calculation, which uses the PARC3D code, solves the three-dimensional Navier-Stokes equations, assuming steady laminar flow on the leading edge region. Experimentally, increases in the spanwise-averaged heat transfer coefficient as high as 50 percent above the two-dimensional value were observed. Numerically, the heat transfer coefficient was seen to increase by as much as 25 percent. In general, under the same flow conditions, the circular leading edge produced a higher heat transfer rate than the elliptical leading edge. As a percentage of the respective two-dimensional values, the circular and elliptical leading edges showed similar sensitivity to spanwise variations in momentum. By equating the root mean square of the amplitude of the spanwise variation in momentum to the turbulence intensity, a qualitative comparison between the present work and turbulent results was possible. It is shown that increases in leading edge heat transfer due to spanwise variations in freestream momentum are comparable to those due to freestream turbulence.
\end{abstract}

\section{Nomenclature}

$\begin{array}{ll}(\rho V) & \text { momentum } \\ a & \text { area } \\ A & \begin{array}{l}\text { peak to peak momentum difference } \\ \text { normalized by the characteristic momentum }\end{array} \\ b & \text { blockage, defined as ratio of maximum }\end{array}$

body thickness to tunnel height

leading edge diameter (twice the leading edge radius of curvature)

Frössling number $\left(F r=N u / \sqrt{R e_{D}}\right)$

heat transfer coefficient $\left(h=q /\left(T_{w}-T_{a w}\right)\right)$

thermal conductivity of fluid

Mach number

Nusselt number $(N u=h D / k)$

pressure

refers to the position 2 diameters

directly above the stagnation point

$\begin{array}{ll}P r & \text { Prandtl number } \\ q & \text { surface heat flux }\end{array}$

$\operatorname{Re}_{D} \quad$ Reynolds number based on leading edge diameter $\left(R e_{D}=\rho V D / \mu\right)$

distance along body surface

temperature

turbulence intensity (also $T u=A_{P} / \sqrt{2}$ )

speed

distance between wire array and

body leading edge

\section{Greek Symbols}

$\gamma \quad$ ratio of specific heats

$\mu \quad$ kinematic viscosity of fluid

$\lambda \quad$ wavelength of spanwise variation

$\rho \quad$ density of fluid

Subscripts and Superscripts

\begin{tabular}{ll}
\hline () & two-dimensional results (i.e., $A=0$ ) \\
()$_{a w}$ & adiabatic wall \\
()$_{c h}$ & characteristic quantity \\
()$_{e d g e}$ & conditions at edge of boundary layer \\
()$_{e x i t}$ & conditions at the exit \\
()$_{i n}$ & conditions at the inlet \\
()$_{s t}$ & conditions at stagnation point \\
()$_{t}$ & total conditions \\
()$_{w}$ & conditions on body (wall) \\
() & spanwise-averaged quantity
\end{tabular}




\section{Introduction}

Accurate prediction of the heat transfer rates on gas turbine blades is very important to the design of efficient and reliable engines. Typically, the leading edge region endures heat transfer rates comparable to the peak value for the entire blade. The leading edge heat, and analogously mass transfer, is known to be sensitive to even low levels of freestream turbulence, and to small amplitude spanwise variations in the freestream momentum $[1,2,3,4,5,6]$. However, the mechanisms responsible for this sensitivity are not well understood. Attempts to explain this sensitivity in terms of a pulsating [7] or oscillating [8] freestream have not produced increases comparable to those seen experimentally. Rigby and VanFossen [9] studied, numerically, the effect of spanwise variation of freestream momentum on the heat transfer to a cylindrical leading edge. They found that stretching of the freestream vorticity introduced by the spanwise variation created highly three-dimensional flow in the leading edge region. This vortex structure near the leading edge was determined to cause large increases in leading edge heat transfer.

It is hoped that a better understanding of the mechanisms which allow low levels of freestream turbulence to cause large increases in stagnation heat transfer can result from the study of a particular isolated phenomenon. The present study addresses the effect of leading edge contour on heat transfer to a body in crossflow. Results with and without spanwise variations in the freestream momentum are discussed. Numerical and experimental results for a cylindrical leading edge and a 3:1 elliptical leading edge are presented. In addition, a specific application of the analysis of Frössling [10] is used to predict the two-dimensional heat transfer to each of the bodies investigated.

\section{Statement of Problem}

Consider the problem of a body with a blunt leading edge. Figure 1 shows, schematically, the geometry of the problem (not drawn to scale). The body is uniform in the spanwise direction and extends far downstream of the leading edge. Let $D$ define the leading edge diameter at the geometric stagnation point. This diameter is defined to be twice the leading edge radius of curvature. The flow is confined on four sides by walls which define the test section. Note that the test section does not vary in width or height in the streamwise direction. The surface of the body is held at a uniform temperature. In the freestream a spanwise variation in momentum is introduced, characterized by a wavelength, $\lambda$, and an amplitude, $A$. The line from $P$ to $P P$ in figure 1 is at the same streamwise location as the leading edge, and is two diameters above the centerline of the tunnel. The amplitude of the spanwise variation of momentum on this line $P-P P$ will be referred to as $A_{P}$. Experimentally, this spanwise variation is produced by a vertical array of wires located upstream of the body. Numerically, the spanwise variation in momentum is specified as a sinusoidal variation at the upstream boundary.

The two geometries to be considered are a circular leading edge and a 3:1 elliptical leading edge. Figure 1 emphasizes the circular leading; the 3:1 elliptical lead-

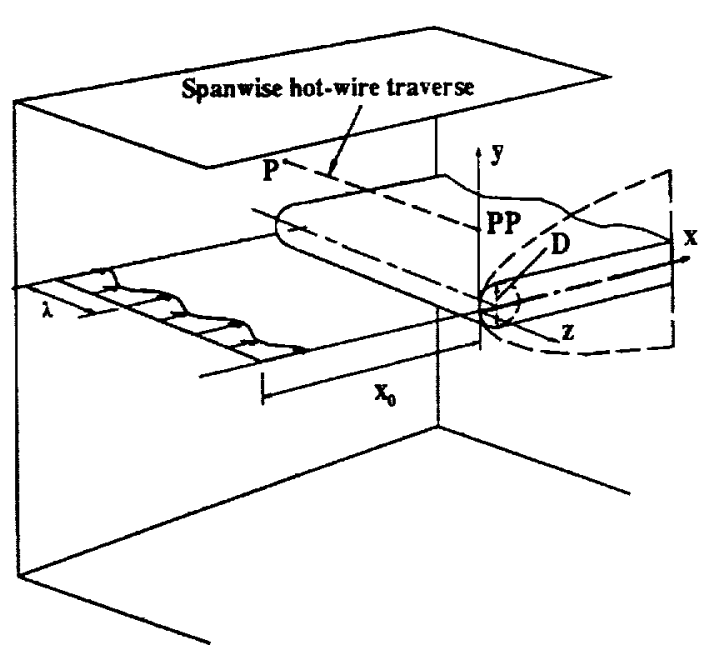

Figure 1: General problem (not drawn to scale).

ing edge is shown as a dashed line for reference. In each case, the leading edge radius of curvature is the same. Thus, for the circular leading edge, the plate thickness is $D$. For the $3: 1$ elliptical leading edge, the plate thickness is $3 D$.

\section{Numerical Method}

To solve this problem numerically, the PARC3D code[11] was used. PARC3D uses the Beam-Warming approximate factorization algorithm and a timemarching implicit scheme which solves the NavierStokes equations in strong conservation form. Some of the basis assumptions used in the PARC3D code are that the fluid behaves as a perfect gas, that the Prandtl number is constant, and that the temperature dependence of the kinematic viscosity is approximated by Sutherland's law [14, p. 328]. PARC3D uses second order accurate central-differencing for spatial derivatives. For stability, fourth difference artificial dissipation is added. The coefficient of the artificial dissipation varies both spatially and directionally, which produces a stable and accurate solution. This form of the artificial dissipation is especially important when computing flows with vorticity in the freestream, since excessive amounts of artificial dissipation can accelerate the decay of vorticity. In the present study, care has been taken to ensure that decay of freestream vorticity is dominated by the physical phenomena, and not by the numerical method. This is done by comparing the rate of decay of vorticity upstream of the body to an analytical result which neglects the presence of the body [9].

The boundary conditions used are as follows. On the body surface, a no-slip condition with uniform temperature is applied. At the inlet, a spanwise total pressure variation is imposed so as to produce a sinusoidal momentum profile, while inlet total temperature is uniform. At the exit, static pressure is specified, while other flow quantities are extrapolated. Symmetry is imposed in the spanwise direction at half wavelength intervals. Thus, only one half wavelength in the spanwise direction is computed. Symmetry is also imposed above and below the body by assuming 
it is at zero angle of attack. Thus, only the top half of the flow field is computed. To take into account the upper wall of the test section, an inviscid wall boundary condition was used. That is, the flow was required to remain tangent to the upper surface with no flow passing through it. No attempt was made to resolve the boundary layer on this upper surface.

It has been shown in a previous study [9] that the PARC3D code can produce accurate solutions to this type of problem. Grids used in the present work are even finer than those that had been shown to be adequate in Rigby and VanFossen [9]. For each case, the residual was required to drop three orders of magnitude from the initial level. The residual is defined to be the sum of the $\mathrm{L} 2$ norms for each of the five equations, divided by the number of grid points. In addition, the value of the Frössling number at the leading edge was monitored. No solution was deemed to have converged until the per iteration change of this Frössling number fell below $10^{-6}$

For the present application, PARC3D was set up to solve the steady laminar three-dimensional flow equations in the leading edge region, the area of interest in this problem. In general, a turbulent solution is required to keep stable any separation which might occur near the junction between leading edge and flat plate. Initiating a turbulent calculation near the junction, while greatly improving the convergence rate, has very little effect on the flow solution in the upstream laminar region. Thus downstream of the leading edge, the algebraic Baldwin-Lomax [12] turbulence model is used.

\section{Experimental Method}

\section{Wind Tunnel}

All tests were conducted in a wind tunnel which was connected to the laboratory exhaust system. This wind tunnel is described in more detail in reference [2]. While operating, room air flowed into a $73.7 \mathrm{~cm}$ wide by $68.6 \mathrm{~cm}$ high rectangular bell mouth past turbulence damping screens and soda straws and then through a $4.85: 1$ contraction. The dimensions of the test section were $15.2 \mathrm{~cm}$ wide by $68.6 \mathrm{~cm}$ high. The maximum velocity obtainable in the test section was $46 \mathrm{~m} / \mathrm{s}$ and the clear tunnel turbulence intensity was less than 0.5 percent at all flow rates. Mass flow rates were measured with an ASME standard sharp edge orifice in the exhaust line; a calibration between mass flow and test section velocity measured in the clear tunnel with a Pitot tube was used to determine test section velocities during runs. For mass flow rates below the capability of the orifice, a hot wire was used to measure test section velocities. Inlet total temperature was measured with four thermocouples just outside the inlet bell mouth.

To establish spanwise variations in momentum, a vertical array of wires perpendicular to the span was placed upstream of the leading edge. The wire array was made up of $0.51 \mathrm{~mm}$ diameter wires spaced 6.3 $\mathrm{mm}$ apart. The amplitude of the spanwise variation reaching the leading edge was varied by changing the streamwise location of the array. The array was positioned from $15.6 \mathrm{~cm}$ to $30.5 \mathrm{~cm}$ upstream of the body leading edge. The position of the wire array relative to the leading edge of the body will be referred to as $x_{0}$. Measurements were also taken with the wire array removed, producing what will be referred to as the two-dimensional results.

\section{Heat Transfer Models}

Spanwise-averaged heat transfer coefficients were measured on a circular leading edge with a $3.30 \mathrm{~cm}$ radius and on a 3:1 elliptical leading edge. The elliptical leading edge design had a semi-major axis of $29.72 \mathrm{~cm}$ and a semi-minor axis of $9.91 \mathrm{~cm}$. Thus the elliptical leading edge had a radius of curvature at the stagnation point of $3.30 \mathrm{~cm}$, which matches the radius of the circular leading edge. Both models spanned the 15.2 $\mathrm{cm}$ wide test section.

Heat transfer coefficients were measured on the surface of the models with electrically heated aluminum strips. Each strip was $6.60 \mathrm{~cm}$ long in the spanwise direction by $4.76 \mathrm{~mm}$ wide in the flow direction and $3.18 \mathrm{~mm}$ deep. A thin film electric heater encapsulated between two layers of polymide plastic was fastened to the back of each aluminum strip with a pressure sensitive adhesive; a thermocouple was then embedded in a groove on the side of each strip. The aluminum heat flux gauges were embedded in the surface of each model with a center-to-center spacing along the surface of about $5.0 \mathrm{~mm}$. The 0.25 $\mathrm{mm}$ wide gap between each heat flux gauge was filled with epoxy. A guard heater was also used behind the surface-mounted heat flux gauges to minimize heat lost to the interior of the model. With the desired flow established, electric current was applied to each gauge individually to keep them all at the same temperature. Heat transfer coefficients for each strip were then calculated from the equation

$$
h=\frac{q_{E I}-q_{\text {rad }}-q_{e n d}-q_{g a p}}{a_{g a g e}\left(T_{g a g e}-T_{a w}\right)}
$$

where $q_{E I}$ is the electric power dissipated in the foil heater, $q_{\text {rad }}$ is the heat lost by radiation from the gauge surface, $q_{\text {end }}$ is the heat lost to the epoxy from the unguarded ends of the aluminum strip, and $q_{g a p}$ is the heat lost to the epoxy gap between gauges. $a_{\text {gage }}$ is the area of the gauge surface exposed to the air, $T_{g a g e}$ is the assumed uniform temperature of the gage measured by the thermocouple, and $T_{a w}$ is the adiabatic wall temperature. The radiation heat loss was estimated using an emissivity of 0.05 for the aluminum. The conduction losses $q_{\text {end }}$ and $q_{g a p}$ were estimated from heat flow in a rectangle (the epoxy) with one side at a constant temperature (the edge of the aluminum), an adjacent side with convection to air, and the other two sides assumed to be insulated $[15, \mathrm{p} .167]$.

\section{Results}

\section{Two-Dimensional Results}

\section{General Remarks}

In this section, cases with no spanwise variation are discussed. One would expect from the 
Frössling analysis [10] that, for two-dimensional flow, the Frössling number results should be nearly independent of Reynolds number. The Frössling number is a dimensionless measure of the heat transfer defined by

$$
F r=\frac{N u}{\sqrt{R e_{D}}}=\frac{\frac{h D}{k_{c h}}}{\sqrt{\frac{(\rho V)_{c h} D}{\mu_{c h}}}}
$$

These two-dimensional results serve two useful purposes. First, they demonstrate the effect of body shape on the heat transfer. Second, the close agreement between experiment, numerical calculation, and analysis lends confidence to the three-dimensional results.

When defining dimensionless quantities such as Reynolds and Frössling numbers, one must decide on the characteristic values of various parameters. Effects such as variable properties, blockage, and compressibility must be addressed. Thus values for these parameters were chosen to maximize the applicability of the results by minimizing the variation in Frössling number from case to case.

Both the experimental and numerical results experience the effects of blockage due to surrounding walls. For internal flow, the choice for characteristic momentum is not immediately apparent. One might choose the inlet value, the exit value, or some other value. For the present study, a simple average between the inlet value and the value in the most blocked region seemed to best account for the effects of blockage. Since the test section geometry does not vary with streamwise distance, the most blocked streamwise position occurs at the thickest part of the body. Thus the blockage can be defined as follows

$$
b=\frac{\text { max. body thickness }}{\text { tunnel height }}
$$

In terms of the inlet momentum, $(\rho V)_{c h}$ can be written as

$$
(\rho V)_{c h}=(\rho V)_{i n} \frac{(2-b)}{2(1-b)}
$$

As mentioned above, this is a simple average between the inlet momentum and the momentum which would occur at the most blocked region due to conservation of mass.

The effect of variable properties appears to be best accounted for by evaluating the viscosity, $\mu$, and the thermal conductivity, $k$, at the freestream total temperature. Evaluating these properties at a film temperature, defined as a simple average between the wall and the total temperatures, was also investigated. It was found that using the film temperature, as opposed to using the total temperature, caused the Frössling number to have a much stronger dependence on temperature difference. This observation was most strongly demonstrated by the numerical results, where cold and hot wall results could be compared. Wall temperatures in the range $0.792 T_{t}<T_{w}<1.188 T_{t}$ were calculated.

It is informative to note the progression of ideas which took place. Preliminary hot wall calculations without blockage were done for the $3: 1$ elliptical leading edge. Using the inlet momentum for the Reynolds number and the freestream total temperature to evaluate properties, it was observed that experimental values of Frössling number were higher than the numerical ones, which agreed well with the Frössling analysis. In an attempt to produce better agreement, the properties were then evaluated at the film temperature, which caused the numerical and experimental Frössling numbers to be reduced producing better agreement between experiment and analysis, but worse agreement between numerical and analytical results. Note that in the analytical results, constant properties are assumed. Experimentally, wall temperatures below the freestream value could not be produced. However, several numerical several runs with negative temperature difference were calculated. For these cases, using film temperature instead of freestream total temperature resulted in Frössling numbers higher than the analytical result. In fact, relative to using the total temperature, the correction from using the film temperature was in the wrong direction.

Finally, it was realized that using the total temperature to evaluate viscosity and thermal conductivity produced nearly the same result for both positive and negative temperature differences. The values were, however, consistently higher than those measured experimentally. At this point, the correction due to blockage was introduced, yielding the desired minimal variation in Frössling number and good agreement for experimental, numerical, and analytical results. Recall that this statement refers to a comparison between numerical results without blockage and the experiment which had blockage of approximately 0.3 for the $3: 1$ elliptical leading edge. At this point it was decided that all numerical calculations would be run with the effects of blockage included. With the blockage nearly matched between the numerical and experimental results, agreement is good with or without the correction for blockage. However, by leaving the effect of blockage in the definition for the characteristic momentum, it is believed that the results presented will more closely correspond to results for unblocked flow.

Eckert and Drake [13, pp. 263-266] suggest that the most appropriate characteristic temperature difference for the evaluation of the heat transfer coefficient $h$ is the difference between the wall temperature and the adiabatic wall temperature. They suggest the adiabatic wall temperature can be approximated by

$$
T_{a w}=\sqrt{\operatorname{Pr}}\left(T_{t}-T_{\text {edge }}\right)+T_{\text {edge }}
$$

The edge temperature is deduced from the surface pressure distribution obtained from the numerical results. Using the isentropic relations, which are valid outside the boundary layer, and assuming that the pressure does not vary across the boundary layer, the edge temperature can be written as

$$
T_{\text {edge }}=T_{t}\left(\frac{p_{w a l l}}{p_{t}}\right)^{(\gamma-1) / \gamma}
$$

\section{Numerical}

Calculations were done using the PARC3D Navier-Stokes code. Boundary conditions and the grid geometry are specified to mimic the experimental facilities. Figure 2 shows the grid for the 3:1 elliptical 
leading edge. As can be seen in figure 2, the grid is one half of a C-type grid. Symmetry was imposed across the stagnation point by assuming a zero angle of attack. The two-dimensional calculations were done on a $48 \times 61 \times 5$ grid. Five grid points are required in the spanwise direction because of the three-dimensional nature of the PARC3D code. These two-dimensional calculations were done with a Reynolds number ranging from 10,000 to 200,000 , and with a wall temperature from $0.792 T_{t}$ to $1.188 T_{t}$. Additionally, the inlet Mach number was varied from 0.1 to 0.2 .

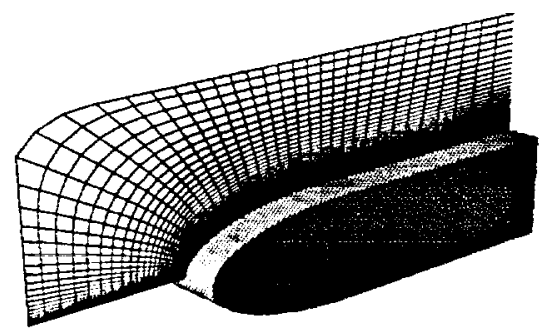

Figure 2: $48 \times 61$ Grid.

Recall that the numerical calculation assumes a constant value for Prandtl number throughout the flowfield. The default value of the Prandtl number in the PARC3D code is 0.72 , and this is the value which was used for all the calculations. For the present experimental results, a Prandtl number of 0.71 is more appropriate. Rather than rerun calculations which had already been done, an adjustment to the Prandtl number of 0.71 is used [16]. All numerical values of the Frössling number are adjusted by assuming that

$$
F r_{0.71}=F r_{0.72}\left(\frac{0.71}{0.72}\right)^{0.4}
$$

Thus, all results presented in this discussion are adjusted to a Prandtl number of 0.71 .

Table 1 provides a summary of the cases run with PARC3D and the resulting Frössling number at the stagnation point. The minimal variation in the stagnation point value of the Frössling number indicates that appropriate decisions have been made for the characteristic quantities. Figure 3 shows the variation of the Frössling number with streamwise distance along the body, for the circular and the $3: 1$ elliptical leading edge. Note that all of the cases listed in table 1 are plotted in figure 3 , which also shows the results of a specific application of the Frössling analysis [10] described in the appendix. The reader is strongly urged to refer to the appendix to see how the Frössling analysis can be applied for specific body contours. Agreement between these numerical results and the Frössling analysis is good.

The minimal variation of Frössling number with increasing Reynolds number indicates that grid resolution normal to the body surface is sufficient for these cases. For the 3:1 elliptical leading edge, there were 17 grid points in the boundary layer at $\operatorname{Re}_{D}=10,100$,

\begin{tabular}{||l|c|c|c|c||}
\hline & $R e_{D}$ & $\frac{T_{w}}{T_{t}}$ & $M_{c h}$ & $\overline{F r}_{s t}$ \\
& & & & \\
\hline elliptical & 10,100 & 1.075 & 0.18 & 0.781 \\
elliptical & 20,300 & 1.075 & 0.18 & 0.766 \\
elliptical & 51,000 & 1.075 & 0.18 & 0.775 \\
elliptical & 101,900 & 1.075 & 0.18 & 0.777 \\
elliptical & 203,800 & 1.075 & 0.18 & 0.778 \\
& & & & \\
circle & 23,500 & 1.074 & 0.21 & 0.939 \\
circle & 23,500 & 1.089 & 0.21 & 0.939 \\
circle & 23,500 & 0.891 & 0.21 & 0.935 \\
circle & 23,500 & 0.911 & 0.21 & 0.936 \\
circle & 23,500 & 0.792 & 0.21 & 0.932 \\
circle & 23,500 & 1.188 & 0.21 & 0.942 \\
circle & 24,600 & 1.082 & 0.11 & 0.939 \\
circle & 24,600 & 0.918 & 0.11 & 0.934 \\
\hline
\end{tabular}

Table 1: Summary of two-dimensional calculations.

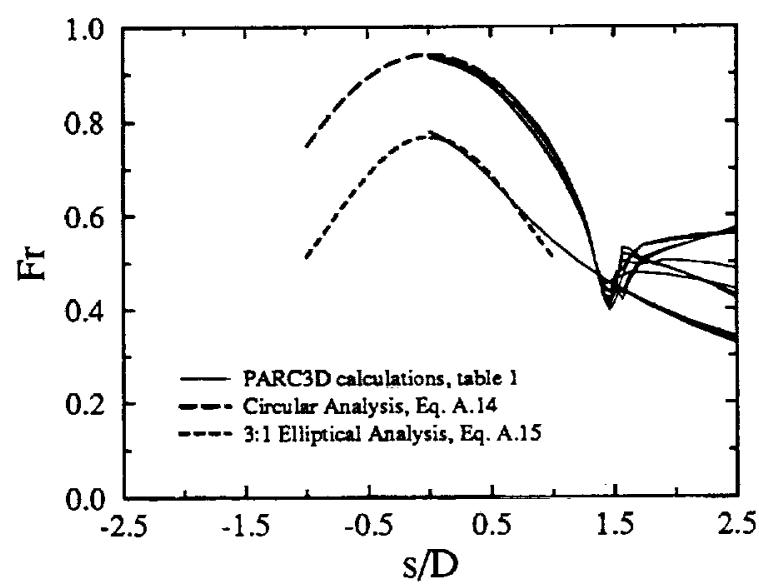

Figure 3: Frössling number vs distance, twodimensional calculations listed in table 1.

and 11 grid points in the boundary layer for $R e_{D}=$ 203,700 . As will be shown below, agreement with experiment is good, which indicates that the streamwise grid resolution is also sufficient for these body shapes.

\section{Experimental}

Experimentally, clear tunnel results were obtained for Reynolds numbers ranging from 77,500 to 232,700 . Wall temperatures on the average of 1.076 times the freestream total temperature are presented. Because the freestream total temperature was the ambient room temperature, approximately $18^{\circ}$ Celsius, the temperature difference was approximately $22^{\circ} \mathrm{Cel}-$ sius above the freestream total. Table 2 shows a summary of the clear tunnel results. The stagnation value of Frössling number is seen to vary little with changes in Reynolds number and temperature difference. Figure 4 shows the variation of Frössling number with distance along the body. This figure shows the re- 
sults from each of the cases shown in table 2. Also on this figure are the results of the PARC3D calculation. Agreement is very good over the measured region. These results are also in good agreement with similar experimental results for a circular cylinder [14, p. 305], and for a 3:1 ellipse [17] in crossflow.

\begin{tabular}{||c|c|c|c||}
\hline & $R e_{D}$ & $\frac{T_{m}}{T_{t}}$ & $\overline{F r}_{s t}$ \\
\hline elliptical & 77,500 & 1.074 & 0.750 \\
elliptical & 108,000 & 1.074 & 0.750 \\
elliptical & 150,900 & 1.074 & 0.770 \\
elliptical & 188,400 & 1.073 & 0.777 \\
elliptical & 232,700 & 1.074 & 0.759 \\
& & & \\
circle & 189,600 & 1.078 & 0.923 \\
circle & 117,400 & 1.077 & 0.927 \\
circle & 151,100 & 1.078 & 0.934 \\
circle & 189,600 & 1.078 & 0.923 \\
\hline
\end{tabular}

Table 2: Summary of two-dimensional experimental results.

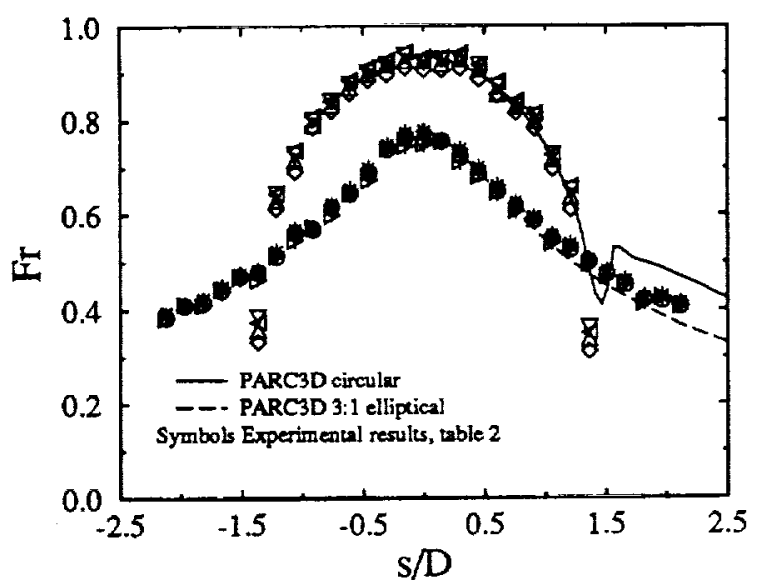

Figure 4: Frössling number vs distance, twodimensional measurements listed in table 2 .

Looking at both figures 3 and 4 it can be seen that agreement between the numerical, experimental, and analytical results is very good. This agreement reinforces the confidence in each of the methods. Also notice that the Frossling number for the 3:1 elliptical leading edge is roughly $20 \%$ lower than for the circular leading edge. The lower value is a direct result of the lower gradient in edge momentum at the stagnation point for the 3:1 elliptical leading edge. The reader is strongly urged to see the appendix for a complete explanation of this statement.

\section{Three-Dimensional Results}

\section{Numerical}

Numerical results were produced for Reynolds numbers from 22,000 to 100,000 . A sinusoidal spanwise profile in momentum was imposed $4.5 \mathrm{D}$ upstream of the leading edge. The wavelength of the spanwise profile was $0.1 D$ for all cases, which matches the wire spacing in the experiment. Cases were run with amplitudes in the range of 1 to $15 \%$ of the freestream momentum. The numerical grid used for the three dimensional runs matched that used for the two-dimensional calculations, except 32 grid points were used in the spanwise direction. The $x-y$ grid was simply stacked in the $z$ direction, equally spaced over one-half wavelength. As in the two-dimensional calculations, symmetry was imposed across the stagnation point. In addition, symmetry was imposed at every half wavelength in the spanwise direction. The Mach number at the inlet for all cases was approximately 0.15 , which was about as low as the PARC3D code could go and still produce reasonable convergence times. No attempt was made to match the Mach number in the experiment since, at these low values, it is not expected to affect the results. Figures 5 and 6 show the variation of spanwise-averaged Frössling number along the body surface, for the circular and elliptical leading edges, respectively. In each figure, results for several amplitudes are shown as well as the result from the Frössling analysis. Recall that the amplitude $A_{P}$ noted in these figures refers to the amplitude of the momentum variation at position $P$ shown in figure 1. The Frössling number was increased above the two-dimensional value for every case, with increases as high as $25 \%$ observed. As a percentage of the corresponding two-dimensional value, the circular and the elliptical leading edges show similar increases in Frössling number for equivalent freestream conditions. Notice that heat transfer is increased quite uniformly for $s / D<0.5$. That is, each curve appears similar to the two-dimensional results, but is shifted up by a certain amount. Note that the spike in figure 5 occurs near the junction between the circle and the flat plate portion of the body. This is also the position where the turbulent solution is initiated. However, no spike is seen in the 3:1 elliptical leading edge results since the junction between the ellipse and the flat plate occurs at $s / D \approx 4$.

\section{Experimental}

Measurements were taken for Reynolds number in the range of 13,000 to 240,000 . The magnitude of the spanwise variation in momentum reaching the body was varied by positioning the array of wires at various streamwise locations. Figures 7 and 8 show the variation of spanwise-averaged Frössling number along the body surface for the circular leading edge and the 3:1 elliptical leading edge, respectively. Increases in the spanwise-averaged Frössling number by as much as $50 \%$ above the two-dimensional value were observed. Introduction of the wires is seen to cause a uniform increase in the heat transfer over most of the measured region. These increases are substantial, and demonstrate the acute sensitivity that the leading edge heat transfer has to small spanwise variations in freestream momentum. 


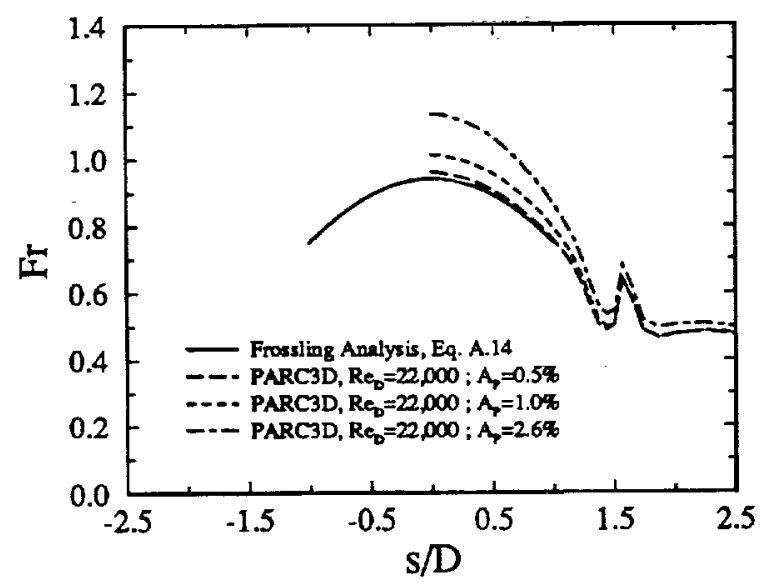

Figure 5: Frössling number vs distance, 3D calculations, circular.

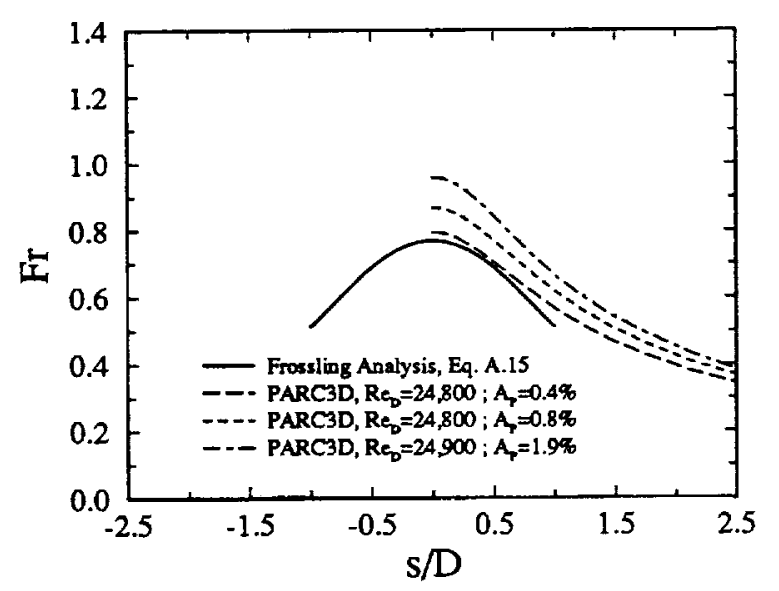

Figure 6: Frössling number vs distance, 3D calculations, elliptical.

With the 3:1 elliptical leading edge model in place and the array of wires located $2.79 D$ upstream of the leading edge, spanwise hot-wire traverses were used to measure the momentum profiles. Figure 9 shows the measured amplitude at position $P$, expressed as a percentage of the reference momentum. Position $P$ is located at the same streamwise location as the leading edge, and approximately two leading edge diameters above the tunnel centerline, see figure 1 . Notice that the amplitude is relatively high at low Reynolds number, then drops off and settles on $4 \%$ over much of the Reynolds number range, perhaps due to mixing of wire wakes. Figure 10 shows the hot-wire trace for one of the largest amplitudes. In this trace, one can see a dominant spanwise variation with spacing equal to the spacing in the array of wires. In addition, there is an unsteady component resulting from low levels of freestream turbulence and unsteadiness in the wire wakes. The amplitude for each trace was arrived at by simply looking at the trace and estimating the average peak-to-peak variation.

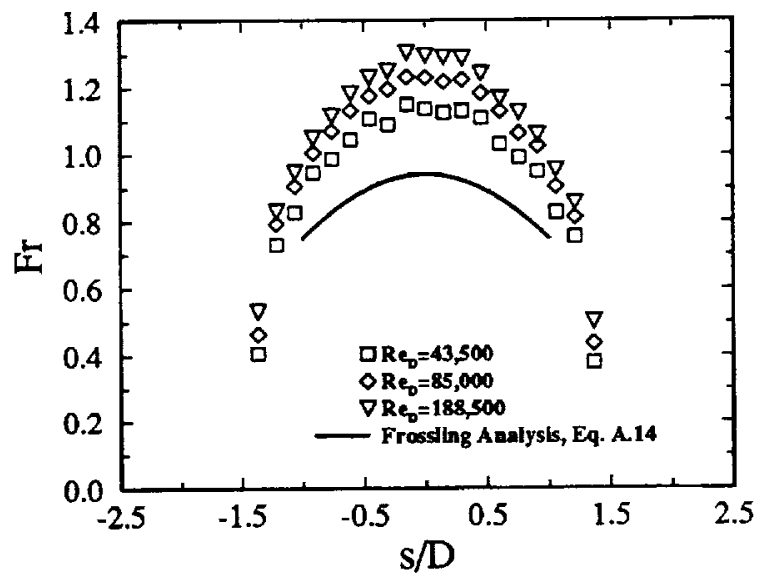

Figure 7: Frössling number vs distance, 3D measured, circular, $x_{0}=2.36 D$.

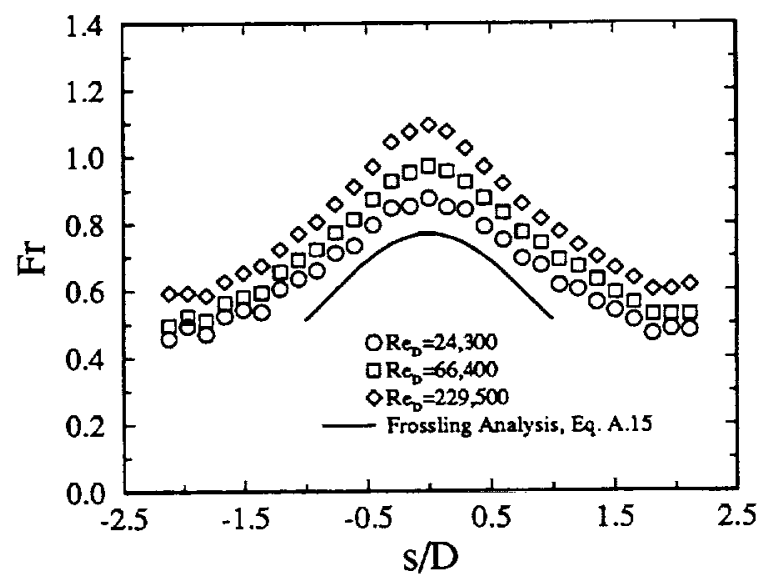

Figure 8: Frössling number vs distance, 3D measured, elliptical, $x_{0}=2.79 D$.

\section{Discussion}

In this section a more direct comparison between measured and calculated heat transfer is presented. It has been demonstrated above that both the numerical and experimental results show a rather uniform increase in Frössling number over the leading edge region. Because of this uniform increase, attention will be focussed on the value at the stagnation point. In addition, each Frössling number is normalized by the appropriate value from the Frössling analysis described in the appendix. For the circular leading edge, the value is 0.9411 . For the elliptical leading edge, the value is 0.7691 .

It is informative to make a qualitative comparison between turbulent results and the present results. To do this, the root mean square value of the amplitude in the present study is equated with the turbulence intensity. Assuming a sinusoidal spanwise variation in momentum, the root mean square value is simply $A_{P} / \sqrt{2}$. As a comparison to turbulent data in general, the correlation of Kestin and Wood [19] is used. While there is much scatter in the heat transfer data in the literature, this correlation does give a qualitative indi- 


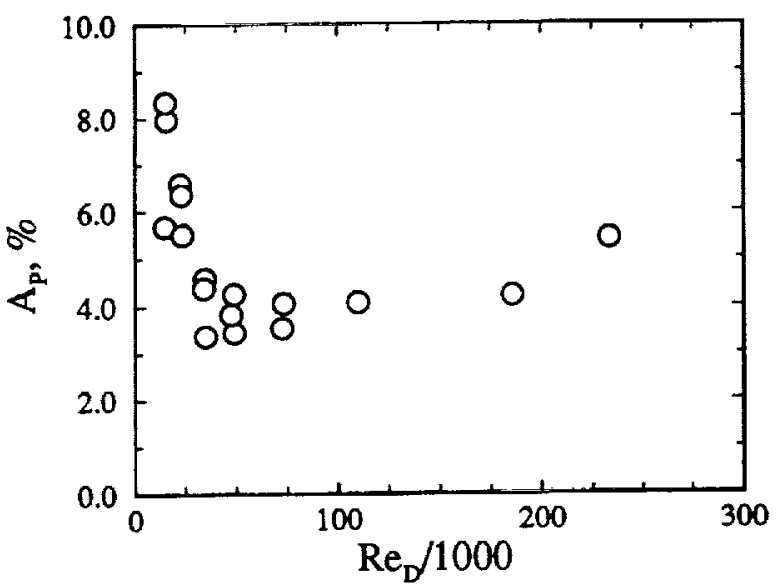

Figure 9: Experimental $A_{P}$ versus Reynolds number, elliptical, $x_{0}=2.79 \mathrm{D}$.

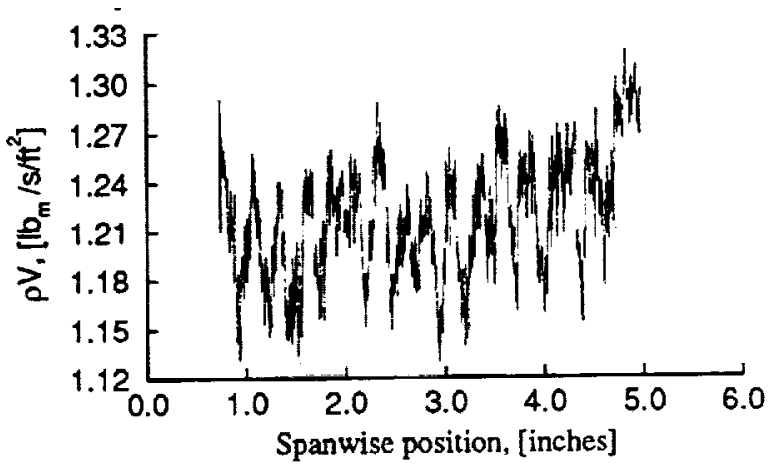

Figure 10: Sample hot-wire trace, elliptical, $x_{0}=$ $2.79 D, R e_{D}=13,000$.

cation of the trend observed in turbulent flow over a circular cylinder. The correlation, normalized by the stagnation point value, is expressed as

$$
F r=1+\frac{3.48}{0.945}\left(\frac{T u \sqrt{R e_{D}}}{100}\right)-\frac{3.99}{0.945}\left(\frac{T u \sqrt{R e_{D}}}{100}\right)^{2}
$$

Figure 11 shows some of the heat transfer results plotted as a function of the quantity $T u \sqrt{R e_{D}}$. Also shown in this figure is the correlation of Kestin and Wood, equation 8 . The experimental results shown in figure 11 are for the $3: 1$ elliptical leading edge, with the array of wires located $2.36 \mathrm{D}$ upstream. This is the only set of measurements for which amplitude levels are available. Notice that the present experimental and the numerical results have a trend similar to the correlation. The exception is that the numerical results seem to level of after an increase of roughly $25 \%$ is achieved. The numerical results indicate a very strong sensitivity at low values of $T u \sqrt{R_{D}}$. However, it would appear that for $T u \sqrt{R e_{D}}>5$, additional flow physics, such as unsteadiness, may be required for direct comparison to experiment. In spite of this, a substantial part of the increase in heat transfer does seem to be accounted for in the numerical results, even at the higher values of $T u \sqrt{R e_{D}}$. Another important observation about the numerical results shown in figure 11 is that the circular and elliptical results plot nearly on top of one another when normalized by their respective two-dimensional values. As will be shown below, the experimental results also exhibit this behavior. If this result is true in general, then knowing the reaction of one body shape to a certain flow could provide information about other body shapes, which could expand the applicability of many existing results. Experimentally, the smallest increase in the Frössling number corresponds to the lowest Reynolds number, while the largest increase occurrs at the highest Reynolds number.

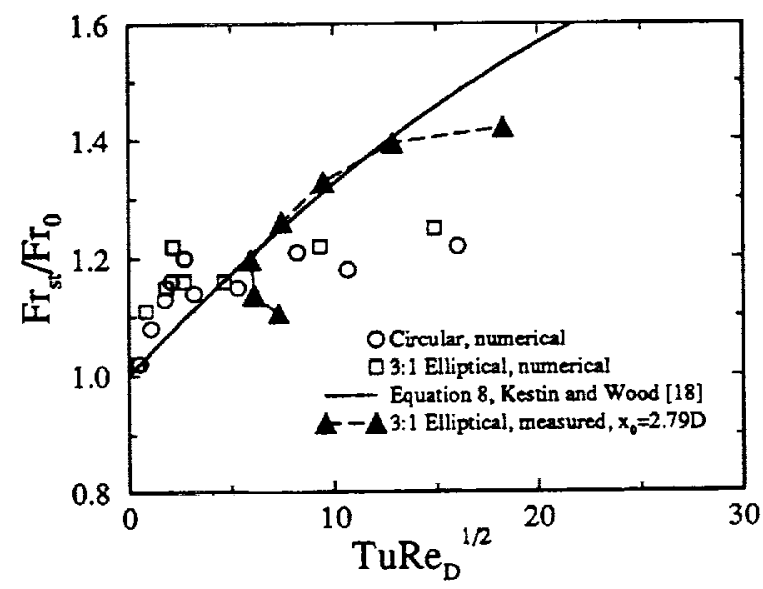

Figure 11: Stagnation Frössling number vs $T u \sqrt{R e_{D}}$.

Figure 12 shows the same data as in figure 11, except plotted as a function of $A_{P}$, the momentum amplitude at position $P$. As stated above, position $P$ is located at the same streamwise location as the leading edge of the body, and approximately two leading edge diameters above the tunnel centerline. This plot demonstrates the very sharp rise in the numerical Frössling number for amplitudes as low as 1 to $2 \%$ of the reference momentum. The numerical results exhibit a much stronger sensitivity to $A_{P}$ than to $R e_{D}$. Due to the number of points on figure 12 , Reynolds numbers are not indicated for each point. However, the relative insensitivity to $R e_{D}$ can be seen by focussing one's attention on the six points clustered above $A_{P}=2 \%$. These six points range in Reynolds number from 22,000 to 100,000 . In fact, the largest increase at $A_{P}=2 \%$ occurred for the lowest Reynolds number. On the other hand, the experimental results exhibit quite a strong effect as Reynolds is varied. Referring once again to figure 12 , one sees that as the Reynolds number increases, the amplitude in the experimental results is fairly constant once it drops off, and yet the heat transfer keeps rising. It has previously been shown [9] that flowfields such as the one studied here may have a spanwise periodic array of vortex structures situated just in front of the stagnation region. These vortex structures are observed in the present calculations. It is observed that as the vortex structure becomes stronger, its position is farther upstream, thus diminishing its ability to increase the heat transfer. While it is observed numerically that the vortex structure becomes stronger with increasing Reynolds number, the previous statement may explain a relative insensitivity of the heat transfer to changes in Reynolds number for the numerical results. 


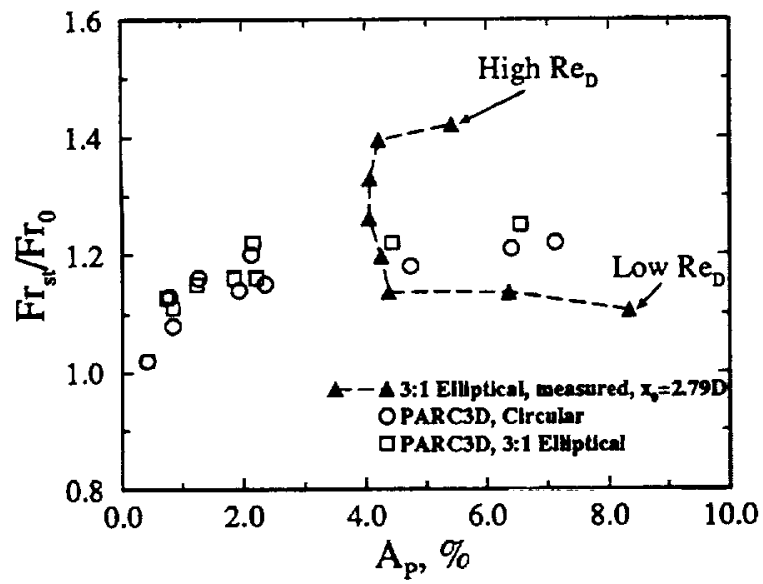

Figure 12: Stagnation Frössling number vs $A_{P}$.

Figures 13 and 14 show the spanwise-averaged stagnation point Frössling number as a function of the square root of the Reynolds number. Also shown in these figures is the correlation of Kestin and Wood, equation 8 . As mentioned above, for the sake of comparison, $T u$ is equated with $A_{P} / \sqrt{2}$. Since the amplitude for the experiment is around $4 \%$ over most of the Reynolds number range, as shown in figure 9 , a value of $T u$ is chosen to be $T u=(0.04) / \sqrt{2}=0.028$. Each result in figures 13 and 14 is normalized by the respective two-dimensional value. The experimental results, figure 14, have a kink near $\operatorname{Re}_{D}=40,000$ (i.e. $200^{2}$ ), then continue to increase as the Reynolds number increases. As was seen in figure 9 , the momentum variation reaching the body initially drops with increasing Reynolds number, then levels off. This drop in amplitude at the lower Reynolds numbers could explain the leveling off seen in the experimental Frössling number at the lower Reynolds numbers. It must also be noted that at the lowest flow rate, the wire wakes appeared to be steady. That is, there was no vortex shedding in the wire wakes. As Reynolds number was increased above 20,000 the wakes became unsteady. Thus, it can not be entirely ruled out that wire wake unsteadiness may be having some effect on these results.

In general, for the numerical results, a larger amplitude produced a larger increase in heat transfer for a given Reynolds number. However, increases of more than about $25 \%$ of the corresponding two-dimensional value does not appear possible for the present calculations. Perhaps additional flow physics, such as unsteadiness, must be included at higher Reynolds numbers. In the calculation of horseshoe vortex flow, Visbal [18] found that a time-periodic solution was possible, even with steady boundary conditions. The present flow situation is similar to the horseshoe vortex problem in some ways. However, the PARC3D code was not run time-accurate, so all solutions presented represent steady flow. Perhaps for an high enough Reynolds number with an high enough amplitude, an unsteady flow for the present geometry could result. The levels required for unsteadiness to occur could not be predicted at this time.

One final and very interesting result shown in figures 13 and 14 is that, when normalized by their respective two-dimensional values, the circular leading

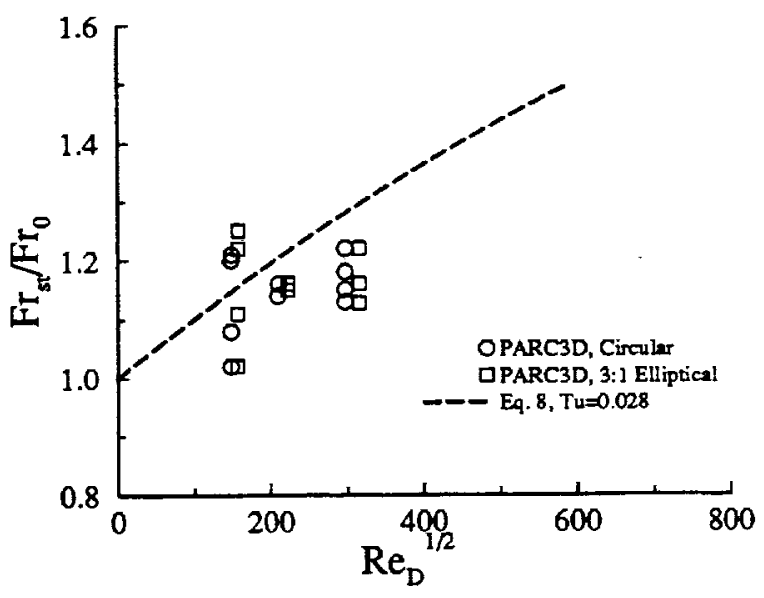

Figure 13: Stagnation Frössling number vs $\sqrt{R e_{D}}$, PARC3D.

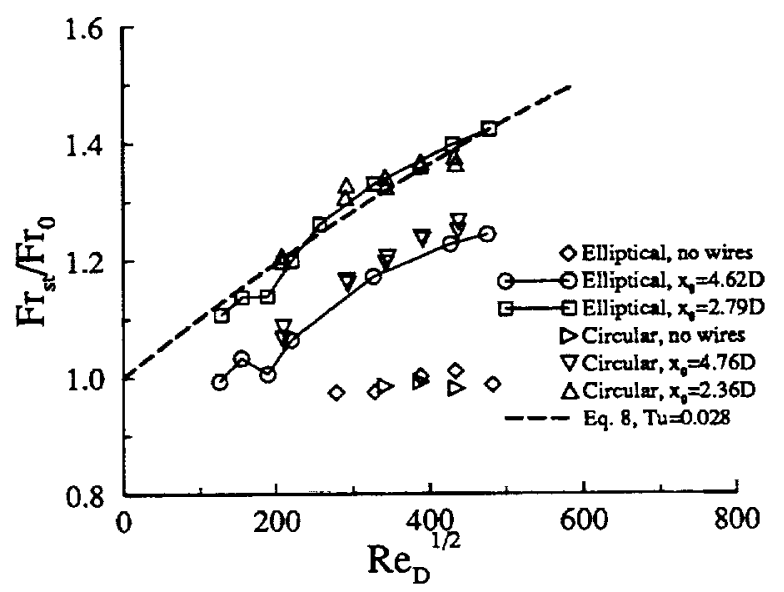

Figure 14: Stagnation Frössling number vs $\sqrt{R \epsilon_{D}}$, Measured.

edge and elliptical leading edge show similar increases in heat transfer for similar flow conditions. This similar increase in heat transfer is seen in both numerical and experimental results. If this result is generally true, then knowing the reaction of one body shape to a certain flow situation could be used to predict the reaction of a second body to the same flow situation.

\section{Conclusions}

It has been shown numerically and experimentally that spanwise variation in the freestream momentum is a very strong mechanism for increasing leading edge heat transfer. For small spanwise variations or for low Reynolds numbers, the numerical results showed increases in heat transfer much like those seen experimentally. However, these three-dimensional steady laminar numerical results were only able to account for at most a 25\% increase in leading edge heat transfer. Experimental results showed increases as high as $50 \%$ above the two-dimensional value. It is felt that increases above $25 \%$ may be due to unsteady effects, such as unsteady wire wakes or time-varying vortex structures near the leading edge. Equating the root 
mean square of the amplitude in the present study to the turbulence intensity of other results in the literature showed qualitative agreement. That is, the leading edge heat transfer showed a similar sensitivity to small steady spanwise variations in freestream momentum, as it did to freestream turbulence.

It was also seen that two bodies with equivalent flow conditions in the freestream experience similar increases in heat transfer, relative to their respective two-dimensional values. These similar increases in heat transfer indicate that heat transfer results for a given body, when normalized by the laminar twodimensional value, could be used to predict the heat transfer to a different body shape under similar flow conditions.

Two-dimensional results were obtained numerically, experimentally, and analytically. Agreement was good for all these results, providing confidence in each of the methods. For equivalent flow conditions, the heat transfer rate to the 3:1 elliptical leading edge was for all cases approximately $20 \%$ lower than that of the circular leading edge. This $20 \%$ difference is seen to be a consequence of the lower gradient in edge velocity at the leading edge for the 3:1 elliptical leading edge. A specific application of the analysis of Frösling is seen to account completely for the differences due to body shape.

One might consider freestream turbulence to be made up of unsteadiness and instantaneous spatial variations in the freestream. By studying the effect of one particular isolated phenomenon, it is hoped that a better understanding of the mechanisms which allow low levels of turbulence to cause large increases in stagnation point heat transfer will result. The work of other researchers, cited herein, suggests that the effect of oscillating or pulsating flow on the stagnation heat transfer is quite small. By contrast, the present study shows that another phenomenon which would exist at any instant in every turbulent flow, namely spanwise variations, can cause large increases in leading edge heat transfer. Thus further study of this mechanism appears warranted, in particular, the combination of spanwise variations and unsteadiness.

\section{Acknowledgements}

The present work was supported by the NASA Lewis Research Center under contract NAS3-25266 with G.J. Van Fossen as monitor. Calculations were done on both the CRAY/YMP at Lewis and at NAS.

\section{References}

[1] J. Kestin and R. T. Wood. Enhancement of stagnation-line heat transfer by turbulence. Progress in Heat Mass Transfer, 2:pp. 249-253, 1969 .

[2] G. J. VanFossen Jr. and R. J. Simoneau. A study of the relationship between free-stream turbulence and stagnation region heat transfer. ASME J. Heat Transfer, 109:pp. 10-15, 1987.
[3] P. R. Hodson and H. M. Nagib. Vortices induced in a stagnation region by wakes: Their incipient formation and effects on heat transfer from cylinders. paper 77-790, AIAA, June 1977.

[4] V. Kottke. On the visualization of longitudinal vortices in stagnation flows. In Flow Visualization $I V$, pages 321-326. Hemisphere Publishing Corp., 1986.

[5] V. Kottke and B. Mpourdis. On the existence of Taylor-Görtler-vortices on concave walls. In Flow Visualization IV, pages 475-480. Hemisphere Publishing Corp., 1986.

[6] M. L. Marziale and R. E. Mayle. Mass transfer from a circular cylinder: Effects of flow unsteadiness and 'slight nonuniformities'. Technical Report CR-174759, NASA, Sept 1984. Rensselaer Polytechnic Institute, Troy, New York.

[7] M.J. Lighthill. The response of laminar skin friction and heat transfer to flucuations in the stream velocity. Proc. Roy. Soc. Series A, 224, No. 1:pp. $1-23,1954$.

[8] E.P. Childs and R.E. Mayle. Heat transfer on a rotationally oscillating cylinder in crossflow. Int. J. Heat Mass Transfer, 27, No. 1:pp. 85-94, 1984.

[9] D.L. Rigby and G.J. Van Fossen. Increased heat transfer to a cylindrical leading edge due to spanwise velocity variations in the freestream. paper 91-1739, AIAA, June 1991.

[10] N. Frössling. Evaporation, heat transfer, and velocity distribution in two-dimensional and rotationally symmetrical laminar boundary-layer flow. Technical Report TM-1432, NACA, 1940.

[11] G. Cooper and J. Sirbaugh. The PARC distinction: A practical flow simulator. paper 90-2002, AIAA, July 1990.

[12] B. S. Baldwin and H. Lomax. Thin layer approximation and algebraic model for separated turbulent flows. paper 78-257, AIAA, 1978.

[13] E.R.G. Eckert and Jr. R.M. Drake. Heat and Mass Transfer. McGraw-Hill Book Company, Inc, 1959.

[14] H. Schlichting. Boundary-Layer Theory. McGraw-Hill, Inc., 1979. Seventh Ed.

[15] H. S. Carslaw and J. C. Jeager. Conduction of Heat in Solids. Clarendon Press, Oxford, 1959. Second Ed.

[16] S. Goldstein. Modern Developments in Fluid Dynamics. Oxford Univ. Press, 1938.

[17] R.M. Drake, R.A Seban, D.L. Doughty, , and S. Levy. Local heat-transfer coefficients on surface of an elliptical cylinder, axis ratio $3: 1$, in a highspeed air stream. Trans. ASME, 75, No. 7 :pp. 1291-1302, 1953.

[18] M. R. Visbal. Numerical Investigation of Laminar Juncture Flows. paper 89-1873 AIAA, June 1989

[19] J. Kestin and R.T. Wood. The influence of turbulence on mass transfer from cylinders. J. of Heat Transfer, 92:pp. 321-327, 1970. 


\section{Appendix}

\section{Frössling Analysis}

The analysis of Frössling [10] is a boundary layer approximation for laminar stagnation point flow, assuming incompressibility and constant properties. The reader is referred to the literature for the details of the analysis; the results are as follows. Let the velocity at the edge of the boundary layer, normalized by the freestream value, be approximated by the following

$$
V_{e d g e}=V_{1}\left(\frac{s}{D}\right)+V_{3}\left(\frac{s}{D}\right)^{3}+V_{5}\left(\frac{s}{D}\right)^{5}
$$

where $s$ is the distance measured along the surface, $D$ is the leading edge diameter, and $V_{1}, V_{3}$, and $V_{5}$ are constants which depend on body shape. The Frössling number distribution can then be expressed as

$$
F r=F_{0}+F_{2}\left(\frac{s}{D}\right)^{2}+F_{4}\left(\frac{s}{D}\right)^{4}
$$

where

$$
\begin{aligned}
& F_{0}=-f_{0} \sqrt{2 V_{1}} \\
& F_{2}=-\frac{8 V_{3} f_{2}}{\sqrt{2 V_{1}}} \\
& F_{4}=-\frac{12 V_{5}}{\sqrt{2 V_{1}}}\left(g_{4}+\frac{V_{3}^{2}}{V_{1} V_{5}} h_{4}\right)
\end{aligned}
$$

The coefficients $f_{0}, f_{2}, g_{4}, h_{4}$ depend only on Prandtl number. For Prandtl number equal to 0.70 these are [10]

$$
\begin{array}{ll}
f_{0}=-0.4959 & f_{2}=-0.1119 \\
g_{4}=-0.0977 & h_{4}=0.0318
\end{array}
$$

For air at room temperature, it is more accurate to assume $\operatorname{Pr}=0.71[14, \mathrm{p} .269]$. Rather than recalculate these coefficients for $\operatorname{Pr}=0.71$, it is assumed that

$$
F r_{0.71}=F r_{0.70}\left(\frac{0.71}{0.70}\right)^{0.4}
$$

at each location on the body [16], resulting in approximately a one-half percent adjustment to the Frössling number.

Thus, by specifying the coefficients $V_{1}, V_{3}$, and $V_{5}$ in equation A.1, one can predict the Frössling number distribution in the leading edge region. These coefficients are, in general, dependent on overall body shape. Effect of overall body shape must be taken into consideration for an accurate prediction of the leading edge heat transfer. As will be shown below, the two body shapes considered in the present study, with exactly the same leading edge radius of curvature, can produce stagnation point Frössling numbers which differ by roughly $20 \%$. It will also be shown that this difference can be completely accounted for in terms of the coefficient $V_{1}$ for each body. From these results, it will also be seen that one should not expect a leading edge Frössling number near unity for all body shapes. One must know the gradient of the edge velocity at the leading edge, $V_{1}$ to make an accurate approximation.
For application of the Frössling analysis presented above, a representative case for each body shape was chosen. The edge momentum for each body was deduced from the surface pressure in the numerical calculation. Using the isentropic relations, and assuming constant pressure across the boundary layer, the edge momentum can be written as

$$
(\rho V)_{e d g e}=\sqrt{2 \rho_{\text {exit }}\left(\frac{p_{w a l l}}{p_{\text {exit }}}\right)^{1 / \gamma}\left(p_{t}-p_{w a l l}\right)}
$$

A least squares regression to a fifth order polynomial is then done in the range $0<s / D<1$. The result for the circular leading edge is

$$
\frac{(\rho V)_{e d g e}}{(\rho V)_{c h}}=1.8006\left(\frac{s}{D}\right)-0.4237\left(\frac{s}{D}\right)^{3}+0.0447\left(\frac{s}{D}\right)^{5}
$$

For the 3:1 elliptical leading edge the result is

$$
\frac{(\rho V)_{\text {edge }}}{(\rho V)_{c h}}=1.2027\left(\frac{s}{D}\right)-0.6072\left(\frac{s}{D}\right)^{3}+0.2239\left(\frac{s}{D}\right)^{5}
$$

The edge momentum and the corresponding fifth order polynomial are shown in figure 15 . The fact that equations A. 9 and A.10 pass directly through the numerical results indicates that a fifth order polynomial adequately represents the solution in the range of $0<s / D<1$. Notice that for the 3:1 elliptical leading edge, the gradient at the leading edge, $V_{1}$, is $33 \%$ lower than for the circular leading edge.

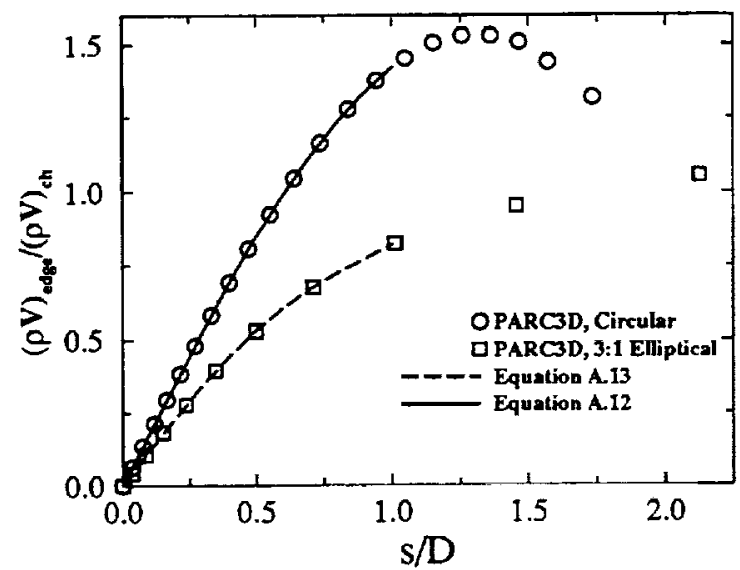

Figure 15: Edge momentum vs streamwise distance.

Putting these values into equations A.2-A.5, and making the correction for Prandtl number equal to 0.71 , equation A.7 gives

$$
F r=0.9411-0.1999\left(\frac{s}{D}\right)^{2}+0.0076\left(\frac{s}{D}\right)^{4}
$$

for the circular leading edge, and

$$
F r=0.7691-0.3505\left(\frac{s}{D}\right)^{2}+0.0938\left(\frac{s}{D}\right)^{4}
$$

for the 3:1 elliptical leading edge. Thus, the Frössling analysis predicts that the Frössling number at the stagnation point will be 0.9411 for the circular leading edge, and 0.7691 for the $3: 1$ elliptical leading edge. 
Public reporting burden for this collection of information is estimated to average 1 hour per response, including the time for reviewing instructions, searching existing data sources, Pathering and maintaining the data needed, and completing and reviewing the collection of information. Send comments regarding this burden estimate or any other aspect of this collection of information, including suggestions for reducing this burden, to Washington Headquarters Services, Directorate for Information Operations and Reports. 1215 Jefler Davis Highway, Suthe 1204, Arfington, VA 22202-4302, and to the Office of Management and Budget. Papenwork Feduction Project (OTO4-0168), Washington, DC, 20503.

\begin{tabular}{l|l|l|} 
1. AGENCY USE ONLY (Leave blank) & 2. REPORT DATE & 3. REPORT TYPE AND DATES COVERED
\end{tabular}

July 1992

\section{TIILE AND SUBTITLE}

dum

Increased Heat Transfer to Elliptical Leading Edges Due to Spanwise Variations in the Freestream Momentum: Numerical and Experimental Results

\section{AUTHOR(S)}

D.L. Rigby and G.J. Van Fossen

7. PERFORIIING ORGANIZATION NAME(S) AND ADDRESS(ES)

National Aeronautics and Space Administration

Lewis Research Center

Cleveland, Ohio 44135-3191
5. FUNDING NUMBEAS

\section{WU-505-62-52}

8. PERFORMING ORGANIZATION REPORT NUMBER

E-7835
9. SPONSORING/MONITORING AGENCY NAME(S) AND ADDRESS(ES)

National Aeronautics and Space Administration

Washington, D.C. 20546-0001
10. SPONSORINGMONITORING AGENCY REPOAT NUMBEA

NASA TM-106150

AIAA-92-3070

\section{SUPPLEMENTARY NOTES}

Prepared for the 28th Joint Propulsion Conference and Exhibit cosponsored by the AIAA, SAE, ASME, and ASEE, Nashville, Tennessee, July $6-8,1992$ D.L. Rigby, Sverdrup Technology, Inc., Lewis Research Center Group, 2001 Aerospace Parkway, Brook Park, Ohio 44142 and G.J. Van Fossen, NASA Lewis Research Center. Responsible person, G.J. Van Fossen, (216) 433-5892.

12a. DISTRIBUTION/AVAILABILTYY STATEMENT

12b. DISTRIBUTION CODE

Unclassified - Unlimited

Subject Category 34

\section{ABSTRACT (Maximum 200 words)}

A study of the effect of spanwise variation in momentum on leading edge heat transfer is discussed. Numerical and experimental results are presented for both a circular leading edge and a 3:1 elliptical leading edge. Reynolds numbers in the range of 10,000 to 240,000 based on leading edge diameter are investigated. The surface of the body is held at a constant uniform temperature. Numerical and experimental results with and without spanwise variations are presented. Direct comparison of the two-dimensional results, that is, with no spanwise variations, to the analytical results of Frossling is very good. The numerical calculation, which uses the PARC3D code, solves the three-dimensional Navier-Stokes equations, assuming steady laminar flow on the leading edge region. Experimentally, increases in the spanwise-averaged heat transfer coefficient as high as 50 percent above the two-dimensional value were observed. Numerically, the heat transfer coefficient was seen to increase by as much as 25 percent. In general, under the same flow conditions, the circular leading edge produced a higher heat transfer rate than the elliptical leading edge. As a percentage of the respective two-dimensional values, the circular and elliptical leading edges showed similar sensitivity to spanwise variations in momentum. By equating the root mean square of the amplitude of the spanwise variation in momentum to the turbulence intensity, a qualitative comparison between the present work and turbulent results was possible. It is shown that increases in leading edge heat transfer due to spanwise variations in freestream momentum are comparable to those due to freestream turbulence.

\section{SUBJECT TERMS}

Heat transfer; Stagnation point; Elliptical leading edge; Three dimensional; Numerical experimental

17. SECURITY CLASSIFICATION OF REPORT

Unclassified

18. SECURIT CLASSIFICATION
OF THIS PAGE
Unclassified

Unclassified
19. SECURTY CLASSIFICATION OF ABSTRACT Unclassified 